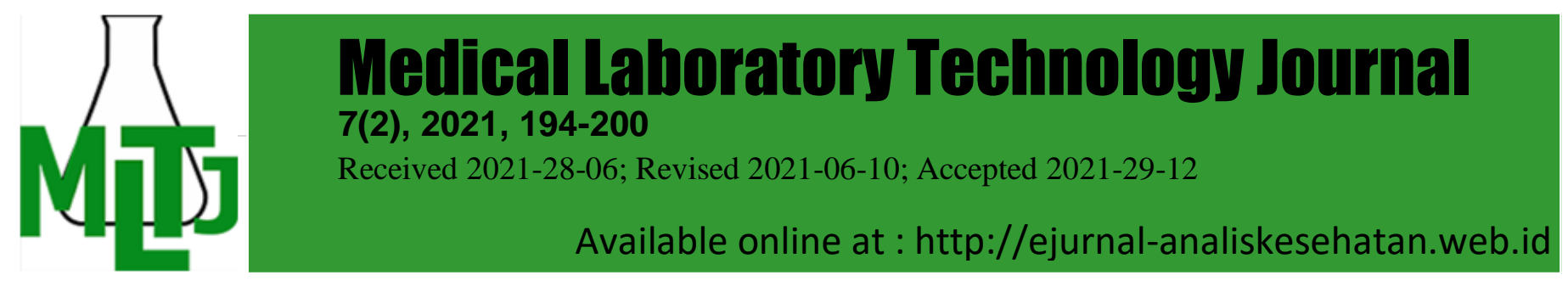

\title{
Patients that are having Covid-19 Disease After the First Dose of Covid-Vaccine
}

\author{
Hakan S. Sayiner ${ }^{1 *}$, Aski Vural ${ }^{2}$, Fatih Dogan ${ }^{3}$, Sedat Parlak ${ }^{2}$, Furkan Bakirhan ${ }^{4}$ \\ ${ }^{1}$ Department of Clinical Infectious Diseases and Medical Microbiology, Faculty of \\ Medicine, Adıyaman University, Training and Research Hospital, Turkey. \\ ${ }^{2}$ Department of Internal Medicine, Faculty of Medicine, Adıyaman University, \\ Training and Research Hospital, Turkey. ${ }^{3}$ Department of Plastic and Reconstructive \\ Surgery, Faculty of Medicine, Adıyaman University, Training and Research Hospital, \\ Turkey. ${ }^{4}$ Department of Public Health, Faculty of Medicine, Adıyaman University, \\ Turkey. *Email: drhssayiner@yahoo.com. DOI: 10.31964/mltj.v7i2.390
}

\begin{abstract}
There is no effective treatment for the Covid-19 disease that is still impacting the whole world. It considered that vaccines, one of the effective means to prevent infectious diseases, will play a significant role in protecting from the Covid-19 Disease. Vaccinations generals performed before exposure. We aimed to follow the clinical and laboratory progress of patients who got Covid-19 after the first dose of the Covid-19 vaccine and to evaluate the changes that may occur in antibody formation. 13 patients, who received the first dose of COVID 19 vaccine Coronovac as of 14.01.2021 when vaccination started in Adıyaman University Training and Research Hospital/Turkey, who had symptoms after the vaccine and whose real-time PCR found to be positive, were included in the study. After the first dose of the Covid-19 vaccine, 13 patients exam. Three of our patients were female, and 10 of them were male. The average age was 38.8. British variant detected in 4 of our patients; 1 of them was female. Contact times differed between 3 and 5 days. The most common symptoms were muscle-joint pain (53.8), weakness-fatigue (46.2), fever (38.5), and headache (30.8). Three of our patients, being one female, received inpatient treatment. Furthermore, our five patients who checked regarding Sars cov-2 IgG became positive in the first month. No adverse changes in the course of the illness observed in the patients were RT-PCR positive after the first dose of the Covid-19 vaccine. Antibody response detected at the end of the first month. Vaccination is an effective method for taking infectious diseases under control but adhering to personal protective measures still maintains their importance.
\end{abstract}

Keyword: Covid-19 disease; Covid-19 vaccine; Reverse Transcription-Polymerase Chain Reaction (RT-PCR)

\section{INTRODUCTION}

The Covid-19 disease caused by the Sars Cov-2 virus, which impacted the whole world starting from the first months of 2020, causing a pandemic, was first demonstrated in December 2019 (Jin et al., 2020). Symptoms such as fever, cough, difficulty breathing, headache, and muscle-joint pain are the symptoms of Covid-19. (Xu et al., 2020)

No complete and effective treatment has been founded for this new virus, and related efforts are still ongoing for this purpose (Bhimraj et al., 2019; Bhimraj et al., 2020). Developing vaccines, one of the most effective ways to prevent diseases, may become the most crucial approach to prevent the Sars Cov-2 virus from causing 
COVID-19. This way will play a vital role in taking the spread of the virus under control and decreasing the death rate (Dong et al., 2020). New Vaccination before contact is still essential in preventing infectious diseases (Rawat et al., 2021). Pre-exposure vaccination protects those not infected from infection, while post-exposure vaccination may change or control the clinical course of becoming ill among the infected ones (Mortimer, 2003).

Studies conducted on the efficacy and safety of vaccines used against Covid19 disease (Tanriover et al., 2021, Soiza et al. 2020) and post-vaccine side effects (Onsun et al., 2021); however, there are no studies on the clinical and laboratory follow-up of people who got Covid-19 disease after vaccination and on the interaction with drugs used against the disease. This study presented to follow the clinical and laboratory progress of patients who showed symptoms of Covid-19 illness after the first dose of the Covid-19 vaccine and whose PCR test was positive and to draw attention to the interaction with drugs used against covid-19 disease and its effects on antibody formation.

\section{MATERIALS AND METHODS}

In this study, we examined the clinical findings, hospitalization rates, some laboratory parameters, lung involvement, and antibody levels of our 13 patients who had complaints such as fever, weakness, cough, headache, joint pain and who were diagnosed with Covid-19 RT-PCR (PCR ROCHE BIO-RAD Kiagen device, RTA kit) and mutation-positive before getting the second dose of the vaccine subsequent of getting the first dose of inactivated COVID-19 vaccine, Sinovac CoronaVac. Coronovac after the date of 14.01.2021 (Table1-Table 3). In this study, approval was obtained from the presidency of Biomedical Research Ethics Committee, Medical Faculty, Adıyaman University, Adıyaman, Turkey, with approval code 2021/03-12 on $16 / 03 / 2021$. Antibody study quantitatively carried out in the GRIFOLS branded TIRITURUS model device with micro-ELISA method after $5 \mathrm{cc}$ of blood transferred into the biochemistry tube was centrifuged.

\section{RESULTS AND DISCUSSION}

Following the first dose vaccine, 13 individuals identified with the symptoms of COVID-19 disease and PCR positivity. The average age of the patients was 38.84 years, ranging from 24 to 53 years. While $3(23 \%)$ of them were female, $10(76 \%)$ were male. British variant detected in $4(30 \%)$ patients, and one was female. Two of our patients had a contact history with their relatives, whereas the other two patients had a contact history with a Covid-19 patient; their contact times ranged from 3 to 5 days. The most common symptoms seen in our patients were muscle-joint pain (53.8), weakness-fatigue (46.2), fever (38.5), and headache (30.8). Again the British variant was positive in three patients having cough-the demographic and clinical characteristics of the patients given in Table 1 .

Five of our patients find to have lung involvement. Three of them, one female, had inpatient treatment, and their length of stay ranged between 4 and 6 days. In the blood parameters of our patients, WBC levels was found to be within normal limits, and lymphocyte levels of 3 patients were detected to be below 10 percent. C-reactive protein (CRP) levels were between 1.1 and $13.4 \mathrm{mg} / \mathrm{dl}$ in 6 patients. Ferritin level was $1500 \mathrm{ng} / \mathrm{ml}$ in 1 patient, while it was normal in 3 patients examined. The D-dimer level was identified to be $750 \mu \mathrm{g} / \mathrm{L}$ in 1 patient. It was observed that procalcitonin levels changed between 0.13 and $0.16 \mathrm{ng} / \mathrm{mL}$ in 4 of our patients. 
Table 1: Demographic, Clinical, and Laboratory Findings of The Patients Mutation

Existing

Not existing

Complaint beginning

after vaccination (hours)

RT-PCR positivity after

vaccination - which day

PCR negativity - which

day

$4 \quad 30,8 \%$

$9 \quad 69,2 \%$

$230,92 \pm 231,80$

120(2-720)

$11,84 \pm 8,88$

$7(2-28)$

$10,33 \pm 3,57$

\section{Symptoms}

Muscle-joint pain $11(4-15)$

Weakness fatigue

7

Fever

Headache

Sore throat

Cough

7

53,8

Shivering

Nasal Flow

$6 \quad 46,2$

$5 \quad 38,5$

$4 \quad 30,8$

$3 \quad 23,1$

$3 \quad 23,1$

Backache

$2 \quad 15,4$

Loss of taste smell

Sweating

$1 \quad 7,7$

$1 \quad 7,7$

$1 \quad 7,7$

Hospitalization

\begin{tabular}{lcc} 
Existing & 3 & 23,1 \\
Not Existing & 10 & 76,9 \\
\hline
\end{tabular}

\section{Laboratory}

Wbc

$\left(10^{3} \mu \mathrm{L}\right)$

Lym\%

$6,02 \pm 1,63$

$5,55(4,10-8,90)$

$20,52 \pm 9,24$

$20,9(5,4-36,7)$

Lym

$1,15 \pm 0,49$

$\left(10^{3} \mu \mathrm{L}\right)$

$1,2(0,30-1,9)$

CRP

$3,26 \pm 4,36$

$(\mathrm{mg} / \mathrm{dL})$

$1,3(0,2-13,4)$

Ferritin

(ng/ml)

$425,1 \pm 609,06$

D-dimer

206(53,2-1500)

$(\mu \mathrm{g} / \mathrm{L})$

$345,8 \pm 176,12$

Prc 304,5(180-750)

(ng/mL)

$0,13 \pm 0,01$

\section{Treatment}

$0,13(0,12-0,16)$

Those not received

Those received

Levofloxacin +dmah

Favi+Levofloxacin

Favipiravir+Levofloxacin

7

6

1

3

2

+steroid+dmah

Wbc: White blood cell, Lym: lymphocyte, Lym\%: lymphocyte

${ }^{*} 100 /$ wbc, CRP: c-reactive protein, Prc: procalcitonin

53,8

46,2

7,7

23,1

15,4 
Three of our patients received favipiravir, levofloxacin, low molecular weight heparin (dmah) treatment, whereas 2 of them took favipiravir levofloxacin, dmah, and steroid. One of our patients received levofloxacin and dmah treatment. Seven of our patients received no therapy (Table-1). While 1 of our five patients, whose CT findings complied with covid, was PCR positive in the 1st week after the vaccine, the other four patients were positive within the timeframe after the 1st week. The involvement detected in CT was mild.

PCR test with the purpose of control performs on 12 patients. The PCR turned negative between 4 and 15 days with an average of 9.25 days in the patients without mutated virus and between 11 and 15 days with an average of 12.25 days in patients with the mutated virus. Antibody levels were measured in 3 patients in the first week. $\lg G$ and $\lg M$ find to be negative. In the 2nd week, one patient was determined to have IgG and IgM negative, whereas two patients were IgM negative and IgG positive. In the 4th week, five patients were identified with having IgG positive (detected between 48.9 and 61), one patient had IgM negative, and four patients were found to have it positive (between 7.9 and 45) (Table-2).

\begin{tabular}{|c|c|c|c|c|c|c|}
\hline \multirow{2}{*}{$\begin{array}{l}\text { Patient } \\
\text { No. }\end{array}$} & \multirow{2}{*}{$\begin{array}{l}\text { PCR } \\
\text { negativity }\end{array}$} & \multirow[t]{2}{*}{$1^{\text {st }}$ week } & \multicolumn{2}{|c|}{$2^{\text {nd }}$ week } & \multicolumn{2}{|c|}{$4^{\text {th }}$ week } \\
\hline & & & $\lg G$ & $\lg M$ & $\lg G$ & $\lg M$ \\
\hline 1 & 6 & $5,8(-)^{*}$ & & & & \\
\hline 2 & 8 & $(-)$ & $9,4(+)$ & $4,8(-)$ & $48,9(+)$ & $2,6(-)$ \\
\hline 3 & 4 & $(-)$ & $39(+)$ & $4,8(-)$ & & \\
\hline 4 & 6 & & $(-)$ & $(-)$ & $61(+)$ & $16,7(+)$ \\
\hline 5 & 11 & & & & & \\
\hline 6 & 12 & & & & & \\
\hline 7 & 12 & & & & & \\
\hline 8 & 15 & & & & & \\
\hline 9 & 11 & & & & $54,4(+)$ & $45(+)$ \\
\hline 10 & 11 & & & & $59,5(+)$ & $29,2(+)$ \\
\hline 11 & 13 & & & & $59,5(+)$ & $7,9(+)$ \\
\hline 12 & $\begin{array}{l}\text { Control PCR } \\
\text { not available }\end{array}$ & & & & & \\
\hline 13 & 15 & & & & & \\
\hline
\end{tabular}

After the first dose of vaccination against the Covid-19 disease, 13 people have become ill due to the Covid-19. Various symptoms of the Covid-19 illness was observed in our patients, but none of them needed progression and intensive care. None of our patients follows to have shortness of breath, respiratory distress. CT findings were mild, and three patients received inpatient treatment for observation purposes.

Vaccines have played a role in the prevention of many outbreaks. Pre-exposure vaccination is still vital in taking infectious diseases under control. In addition, it demonstrated that post-exposure vaccination might also be effective, especially in some infections, starting with the rabies vaccine and continuing with the smallpox vaccine. Vaccination aims to protect uninfected individuals from infection before exposure. The immunization performed after direction aims to attenuate or prevent the clinical course of the disease among those infected (Rawat et al., 2021; Mortimer, 2003). 
Table 3: Comparison of Various Findings According to Lung Involvement

\begin{tabular}{lllll}
\hline & & \multicolumn{2}{l}{ Lung Involvement } & Total \\
& & Non-Existing & Existing & \\
\hline Mutation & No & 6 & 3 & 9 \\
\multirow{3}{*}{ Sex } & Yes & 2 & 2 & 4 \\
\multirow{3}{*}{ Treatment } & Male & 6 & 4 & 10 \\
\multirow{3}{*}{ Hospitalization } & Female & 2 & 1 & 3 \\
& Not received & 7 & 0 & 7 \\
& Received & 1 & 5 & 6 \\
\cline { 2 - 5 } Total & No & 8 & 2 & 10 \\
& Yes & 0 & 3 & 3 \\
\hline
\end{tabular}

It was considered that vaccines, to be used against the Covid-19 disease for which no effective treatment has not found, will play a significant role in taking the virus under control and decreasing death rates (Dong et al., 2020). Following up on the patients, who vaccinate after exposure, helped us better understand the effect of vaccines administered and see what they have (Rawat et al., 2021; Mortimer, 2003; Massoudi et al., 2003). No progression was observed in the clinic of 7 patients who caught the illness in the first week of vaccination. In the CT of one of our patients has been detected a mild involvement.

In a modeling study based on the data obtained from China, the hospitalization rate for COVID-19 increased by age; this reported to be $1 \%$ for people between ages $20,29.4 \%$ for people between ages 50 and 59 , and $18 \%$ for people older than 80 years (Gallagher and Lipsitch, 2019) Two of our patients with no mutation were subjected to inpatient treatment. One patient among the ones with mutation received inpatient treatment. A modeling study carried out by Lauer et al., by utilizing data from 181 confirmed cases with identifiable exposure, estimated that symptoms would develop within 2.2 days in 2.5 percent of infected individuals and within 11.5 days in 97.5 percent of infected individuals. The median incubation period was determined to be 5.1 days (Verity et al., 2020).

In our patients, the median value of the complaint beginning after vaccination ranged from 5 days 2 hours to 30 days. In the previous studies, while the typical clinical findings associated with Covid-19 reports to be fever (88.7\%), cough (67.8\%), fatigue $(38.1 \%)$, sputum $(33.7 \%)$, shortness of breath $(18.7 \%)$, sore throat $(13.9 \%)$ and headache $(13.6 \%)$, the symptoms such as rhinorrhea or nasal congestion, nausea, vomiting, smell and taste disturbances may also use less frequently (Lauer et al., 2020; Guan et al., 2020). The most common symptoms observed in our patients were muscle-joint pain $(53.8 \%)$, weakness, fatigue $(46.2 \%)$, fever $(38.5 \%)$, and headache $(30.8 \%)$. Other symptoms of our patients were identified to be sore throat $(23.1 \%)$, cough $(23 \%)$, and shivering (15.4\%). Cough was seen only in patients detected to have a mutation. One patient finds to have a loss of smell. No patient observes to have shortness of breath.

In research conducted by Long et al. on 285 individuals infected with SARSCoV-2, it has been concluded that $\lg G$ and IgM have increased during the first three weeks after symptom onset and then have begun to decrease (Long et al., 2020). Again in phase II, the clinical trial results of CoronaVac developed by Sinovac showed that neutralizing antibodies extended 14 days after vaccination (Lim et al., 2021). While all of our three patients, whose antibody levels checks, were negative in the 1st week, $\lg G$ antibody levels find to be positive in 2 of our three patients whose antibody 
levels checks in the 2nd week, and in all of our five patients whose antibody levels contained in the 4th week. The lgM levels were positive in 4 of our five patients whose antibody levels check in the 4th week. As a result, we found no delay in the antibody response in those who had the disease after vaccination. It was determined that the antibody response could be earlier than those who only had the disease.

Post-exposure vaccination utilized in the measles epidemic is an effective tool preventing secondary measles cases (Sheppeard et al., 2009). No severe clinical picture was observed in our patients following vaccination, their CT findings have been mild, and IgG antibodies detect at the end of the first month. No patient has had shortness of breath and respiratory distress. The limitation of this study is that since only the Coronovac vaccine administers at the time of the study, the data only belong to this type of vaccine, and the number of patients who got Covid-19 disease after the vaccine was also low.

\section{CONCLUSION}

A complete and effective treatment still has not been found against the Covid 19 disease. The vaccine has been a hope for having protection against the disease. However, recommended personal protection measures should be followed until full immunization by vaccination. In the Covid 19 condition that developed after vaccination, no negative situation was observed due to vaccination; clinical worsening does not see in our patients. We believe that further studies on this matter will provide more insight into whether post-exposure vaccination in Covid-19 disease can likewise contribute to the prevention of disease progression.

\section{ACKNOWLEDGEMENT}

The authors would like to thank all parties involved in this study.

\section{CONFLICT OF INTEREST}

The authors declare that there are no conflicts of interest regarding the publication of this paper.

\section{REFERENCE}

Bhimraj A, Morgan RL, Shumaker AH, Lavergne V, Baden L, Cheng VC, Edwards KM, Gandhi R, Muller WJ, O'Horo JC, Shoham S, Murad MH, Mustafa RA, Sultan S, Falck-Ytter Y. (2020). Infectious Diseases Society of America Guidelines on the Treatment and Management of Patients with COVID-19. Clin Infect Dis. 2020 Apr 27, ciaa478. doi: 10.1093/cid/ciaa478.

Dong, L., Hu, S., \& Gao, J. (2020). Discovering drugs to treat coronavirus disease 2019 (COVID-19). Drug discoveries \& therapeutics, 14(1), 58-60.

Gallagher, T., \& Lipsitch, M. (2019). Postexposure effects of vaccines on infectious diseases. Epidemiologic reviews, 41(1), 13-27.

Guan, W. J., Ni, Z. Y., Hu, Y., Liang, W. H., Ou, C. Q., He, J. X., ... \& Zhong, N. S. (2020). Clinical characteristics of coronavirus disease 2019 in China. New England journal of medicine, 382(18), 1708-1720.

Henderson, D. A., Inglesby Jr, T. V., O'Toole, T., \& Mortimer, P. P. (2003). Can postexposure vaccination against smallpox succeed?. Clinical Infectious Diseases, 36(5), 622-629.

Jin, Z., Du, X., Xu, Y. et al. (2020). Structure of Mpro from SARS-CoV-2 and discovery of its inhibitors. Nature, 582, 289-293. https://doi.org/10.1038/s41586-020-2223y 
Lauer, S. A., Grantz, K. H., Bi, Q., Jones, F. K., Zheng, Q., Meredith, H. R., \& Lessler, J. (2020). The incubation period of coronavirus disease 2019 (COVID-19) from publicly reported confirmed cases: estimation and application. Annals of internal medicine, 172(9), 577-582.

Lee, Y. H., Hong, C. M., Kim, D. H., Lee, T. H., \& Lee, J. (2020). Clinical course of asymptomatic and mildly symptomatic patients with coronavirus disease admitted to community treatment centers, South Korea. Emerging infectious diseases, 26(10), 2346.

Lim, H. X., Lim, J., Jazayeri, S. D., Poppema, S., \& Poh, C. L. (2020). Development of multi-epitope peptide-based vaccines against SARS-CoV-2. Biomedical Journal.

Massoudi, M. S., Barker, L., \& Schwartz, B. (2003). Effectiveness of postexposure vaccination for the prevention of smallpox: results of a delphi analysis. The Journal of infectious diseases, 188(7), 973-976.

Onsun N, Kaya G, Işık BG, Güneş B. (2021). A generalized pustular psoriasis flare after CoronaVac COVID-19 vaccination: Case report. Health Promot Perspect; 11(2),261-262. doi:10.34172/hpp.2021.32

Rawat, K., Kumari, P., \& Saha, L. (2020). COVID-19 vaccine: A recent update in pipeline vaccines, their design and development strategies. European journal of pharmacology, 173751.

Sheppeard, V., Forssman, B., Ferson, M. J., Moreira, C., Campbell-Lloyd, S., Dwyer, D. E., \& McAnulty, J. M. (2009). The effectiveness of prophylaxis for measles contacts in NSW. New South Wales public health bulletin, 20(6), 81-85.

Soiza, R. L., Scicluna, C., \& Thomson, E. C. (2021). Efficacy and safety of COVID-19 vaccines in older people. Age and Ageing, 50(2), 279-283.

Tanriover MD, Doğanay HL, Akova M, Güner HR, Azap A, Akhan S, et al. (2021). Efficacy and safety of an inactivated whole-virion SARS-CoV-2 vaccine (CoronaVac): interim results of a double-blind, randomised, placebo-controlled, phase 3 trial in Turkey. The Lancet, 398(10296), 213-22. Available from: http://www.thelancet.com/article/S014067362101429X/fulltext

Verity, R., Okell, L. C., Dorigatti, I., Winskill, P., Whittaker, C., Imai, N., \& Riley, S. (2019). S.[van Elsland], E. Volz, H. Wang, Y. Wang, X. Xi, CA Donnelly, AC Ghani, NM Ferguson, Estimates of the severity of coronavirus disease, 30243-7.

Xu, X. W., Wu, X. X., Jiang, X. G., Xu, K. J., Ying, L. J., Ma, C. L., \& Li, L. J. (2020). Clinical findings in a group of patients infected with the 2019 novel coronavirus (SARS-Cov-2) outside of Wuhan, China: retrospective case series. bmj, 368. 